\title{
Nonvolatile storage in photorefractive crystals
}

\author{
Demetri Psaltis, Fai Mok, and Hsin-Yu Sidney Li \\ Department of Electrical Engineering, California Institute of Technology, Pasadena, California 91125
}

Received September 2, 1993

\begin{abstract}
We propose and demonstrate a nonvolatile holographic recording system for storing two-dimensional images. The readout light in this system is not absorbed by the holographic medium, and the data are preformatted or postformatted so that lines from different holograms are interleaved to satisfy the Bragg-matching condition.
\end{abstract}

Holographic storage in photorefractive crystals ${ }^{1-3}$ has a high storage density owing to three-dimensional storage and a high readout rate owing to parallel retrieval. The practical development of such memories has been impeded by a lack of materials that have all the suitable properties. Specifically, the most difficult requirement to satisfy is the simultaneous need for materials that are maximally sensitive to light in order to increase the recording speed and the need for a nonvolatile memory that is not affected by illumination to light during readout. Thermal ${ }^{1}$ or electrical ${ }^{4,5}$ fixing are among solutions that were demonstrated to address this problem. Thermal fixing is the most commonly used method now, but it is difficult to design practical systems with this approach. Moreover, it is incompatible with a reprogrammable memory. Electrical fixing is in principle more compatible with a practical reprogrammable memory; however, it is still not well developed. Another approach is the use of periodic copying. ${ }^{6-9}$ With this approach the contents of the hologram are refreshed, preventing decay owing to readout. We can also use materials such as strontium barium niobate, whose absorption varies significantly as the polarization is rotated. ${ }^{10}$ Holograms can then be recorded with one polarization and read out with an orthogonally polarized beam that is less absorbing. This introduces a write/erase asymmetry that can delay significantly the erasure of the holograms.

In this Letter we describe a nonvolatile holographic memory that employs different wavelengths in the recording and readout phases. A dual-wavelength scheme was used previously by McRuer et al. ${ }^{11}$ to implement nonvolatile optical interconnections with a photorefractive crystal. Pauliat et $a .^{12}$ used the dual-wavelength method to construct a deflector. Külich ${ }^{13}$ devised a dual-wavelength storage scheme in which spherical waves are used to construct holograms. In his scheme he could read out a single hologram at a different wavelength by changing the sphericity of the reconstructing wave. In the dualwavelength memory that we describe, plane-wave references are used instead, and multiple holograms can be stored and recalled. The recording wavelength $\lambda_{1}$ is selected near the peak of the trapinduced absorption spectrum of the material. The readout wavelength $\lambda_{2}$ is chosen in a region where absorption is as small as possible. Typically the recording wavelength is shorter than the readout wavelength. The large drop in absorption away from the absorption band yields a very large write/erase asymmetry. For example, we recorded holograms at $\lambda_{1}=488 \mathrm{~nm}$ and exposed the hologram to $\lambda_{2}=$ $632.8 \mathrm{~nm}$ light at a non-Bragg-matched angle for approximately $20 \mathrm{~h}$ at intensities of $0.25 \mathrm{~mW} / \mathrm{cm}^{2}$ without appreciable change in diffraction efficiency.

If a hologram consisting of a single grating is recorded, then the hologram can be Bragg matched at any wavelength if $\lambda_{2}<\Lambda / 2$, where $\Lambda$ is the grating fringe spacing. When the hologram consists of multiple gratings, however, readout becomes more complex, because each grating requires a distinct Bragg-matching condition. We describe a method for circumventing this problem so the information recorded at $\lambda_{1}$ can be read out at $\lambda_{2}$.

The recording geometry is shown in Fig. 1. The information to be stored is presented from a twodimensional spatial light modulator (SLM; a liquidcrystal television in the experiment). Lens L1 takes the Fourier transform of the input image, and a hologram is formed by the introduction of a plane-wave reference beam. Consider first the case in which a single hologram is recorded. The hologram is equivalent to a superposition of sinusoidal gratings, with each grating corresponding to a pixel on the SLM. We would like the readout beam to be simultaneously Bragg matched to all the gratings so that the entire recorded hologram can be efficiently reconstructed by the $\lambda_{2}$ beam. Unfortunately this is not possible. We explain this with the aid of the $k$-space diagram shown in Fig. 2. The recording reference beam is drawn as a single vector, $\mathbf{R}_{1}$, whereas the signal beam coming from the SLM is drawn as a cone of $k$ vectors. A representative wave vector, $\mathbf{S}_{1}$, is shown in Fig. 2. The hologram itself consists of grating vectors connecting the tip of the reference $k$ vector to all the points of the signal cone $k$ vectors on the $\lambda_{1}$ $k$ sphere. The grating vectors are not drawn in Fig. 2. To consider what happens when we illuminate the hologram with a $\lambda_{2}$ plane wave, we need to draw a second $\lambda_{2} k$ sphere, which we assumed to be smaller in Fig. 2. This plane wave is represented by the $\mathbf{R}_{2}$ wave vector in Fig. 2. For clarity we have assumed that the hologram is illuminated by the $\lambda_{2}$ beam from the opposite side, but the hologram can be read out from either side. The intersection of the 


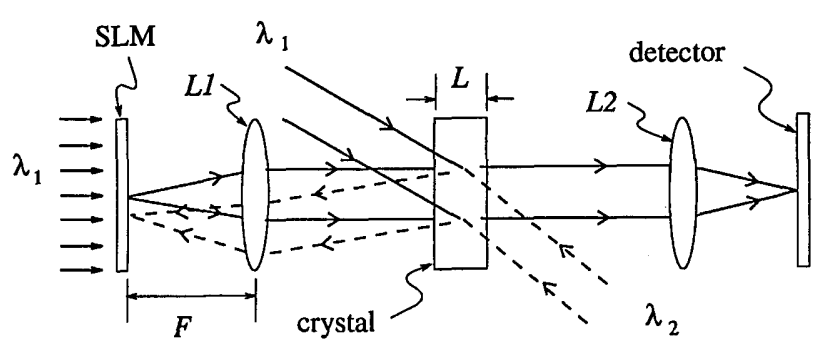

Fig. 1. Setup for recording and retrieving twodimensional images by use of two wavelengths. L2, imaging lens.

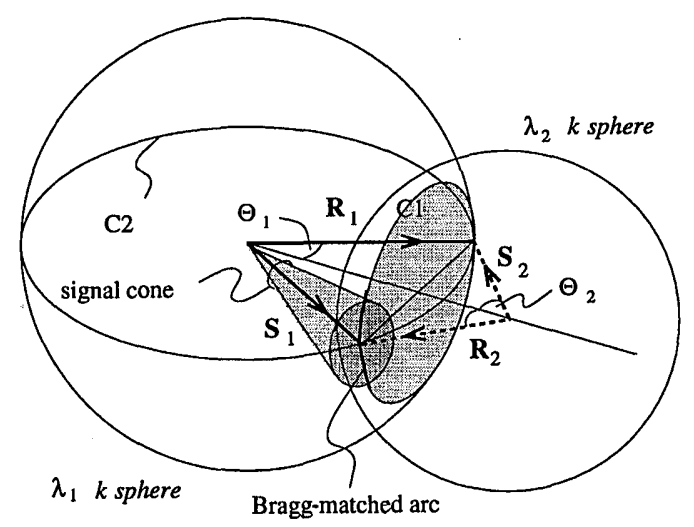

Fig. 2. $k$-space diagram of the Bragg-matching condition in the two-wavelength system.

two spheres forms a circle, $\mathrm{C} 1$. A grating recorded at $\lambda_{1}$ is Bragg matched to the $\lambda_{2}$ readout beam if its tip lies on the intersecting circle, C1. As Fig. 2 shows, the signal cone intersects the circle only along a short arc. Therefore only those pixels on the SLM whose corresponding wave vectors fall on this arc are Bragg matched. In the paraxial approximation the arc can be approximated by a line. If the crystal is sufficiently thick, only one vertical line of pixels on the SLM will be within the Bragg-matched regime. The thickness $L$ that ensures that only one line is reconstructed is

$$
L=\frac{2 \lambda_{1} F}{\Delta x_{w}} \frac{1}{\tan \left(\theta_{2}-\theta_{1}\right)},
$$

where $F$ is the focal length of the lens $L 1$ and $\Delta x_{w}$ is the width of the pixel on the SLM. $2 \theta_{1}$ is the angle (inside the crystal) between the recording reference beam and the plane wave in the signal beam corresponding to the pixel that is exactly Bragg matched when read out with $\lambda_{2} . \quad 2 \theta_{2}$ is the angle (inside the crystal) between the readout reference beam and the reconstructed signal plane wave that corresponds to a pixel that is exactly Bragg matched (Fig. 2). It is assumed that the signal beam is at normal incidence at the crystal.

The position of the reconstructed line is determined by $\theta_{2}$. $\theta_{2}$ is in turn determined by $\theta_{1}, \lambda_{2}$, and $\lambda_{1}$ from the geometry of Fig. 2:

$$
\frac{\sin \theta_{1}}{\lambda_{1}}=\frac{\sin \theta_{2}}{\lambda_{2}}
$$

Note that, because the angles $\theta_{1}$ and $\theta_{2}$ are not the same, the image reconstructed with $\lambda_{2}$ is not the same as the original position on the SLM during recording.

Now suppose that, instead of a single image, multiple images are angularly multiplexed at $\lambda_{1}$. When the recorded hologram is illuminated with a plane wave at $\lambda_{2}$, a single line of each stored image is Bragg matched to the illuminating beam. According to Eq. (2), changing the angle of the $\lambda_{1}$ recording beam results in selecting a different line (different $\theta_{1}$ ) from each of the stored images during readout. This is shown in Fig. 3(a), which is a cross-section view of the $k$-space diagram along the plane containing the vectors $\mathbf{R}_{1}$ and $\mathbf{S}_{1}$. We can design the system so that each reconstruction that is due to a $\lambda_{2}$ readout beam is a two-dimensional image whose vertical lines come from different stored images. For example, for the first reconstructed image the first line comes from the first vertical line of stored image \#1, the second line comes from the second vertical line of stored image \#2, and so on, as shown in Fig. 3(b). To ensure that this interleaving is done properly, the following relationship between pixel spacing on the SLM, $\Delta x_{l}$, and the incremental change in the reference beam angle separation during recording, $\Delta \theta_{1 R}$, must hold:

$$
\Delta \theta_{1 R}=\frac{\sin \left(\theta_{2}-\theta_{1}\right)}{\sin \left(\theta_{2}+\theta_{1}\right)} \frac{\Delta x_{l}}{n F},
$$

where $n$ is the index of refraction of the recording material. In Eq. (3), $\Delta \theta_{1 R}$ depends on $\theta_{1}$ and $\theta_{2}$ and therefore on the pixel position. As a result, $\Delta \theta_{1 R}$ cannot be chosen to satisfy Eq. (2) exactly for all pixels. If the angle between the signal and the reference beams is large compared with the angular spread of the signal beam, then this effect can be negligible. Alternatively, a SLM with variable pitch [satisfying Eq. (2)] with a fixed $\Delta \theta_{1 R}$ must be used.

(a)

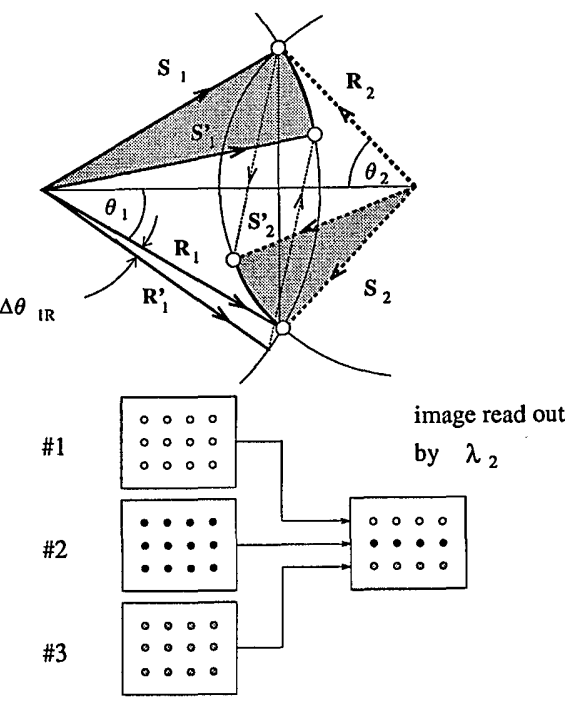

Fig. 3. (a) $k$-space cross-section diagram and (b) interleaving scheme for multiple holograms using the two-wavelength system. The holograms \#1, \#2, and \#3 are recorded at different reference beam angles. On reconstruction with $\lambda_{2}$, only one line from each hologram appears. 
(a)

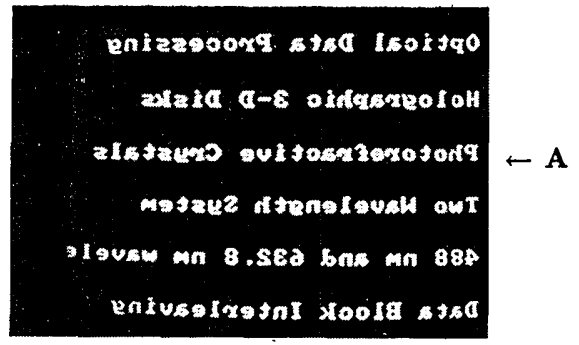

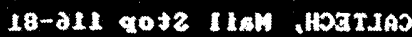

quow ent twenos tsoisgo $\leftarrow B$

totenat ardian kntangd

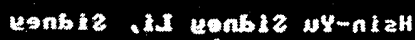

a ston in ing ston is is 9

Enitugmos reatseo ifpat lso

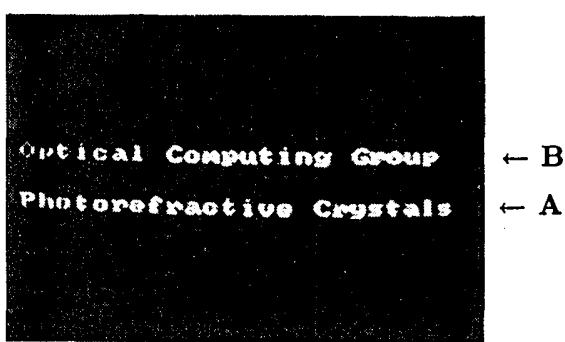

Fig. 4. Experimental results: (a) reconstructed hologram of the first image using the $\lambda_{1}$ plane-wave reference beam, (b) reconstructed hologram of the second image using the $\lambda_{1}$ plane-wave reference beam, (c) reconstructed hologram using $\lambda_{2}$, consisting of two lines from each of the two images. The first line (B) comes from the second image, and the second line (A) comes from the first image.

We performed an experiment to demonstrate the dual-wavelength method, using the setup shown in Fig. 1. The medium used for recording is an irondoped $(0.01 \%)$ lithium niobate crystal purchased from Deltronics, with a thickness of $L=8 \mathrm{~mm}$. The SLM that we used was an Epson liquid-crystal television with $320 \times 320$ pixel resolution (the dimensions are $2.5 \mathrm{~cm} \times 2 \mathrm{~cm}$ ), and the images were generated by a personal computer. The height of the letters is 7 pixels $(0.64 \mathrm{~mm})$, and the separation between the lines is 24 pixels $(2.18 \mathrm{~mm})$. The liquid-crystal television was rotated $90^{\circ}$ so that horizontal lines in the picture were actually vertical in the setup shown in Fig. 1. A CCD camera was used to capture the reconstructed images, which were then displayed on a television monitor, and pictures were then taken off the monitor.

We recorded two angle-multiplexed holograms at approximately $2 \theta_{1}=30^{\circ}$ (outside the crystal), using vertical-polarized $\lambda_{1}=488 \mathrm{~nm}$ light. The separation between the reference beam angles (outside the crystal) is approximately $\Delta \theta_{1 R}=7 \times 10^{-4} \mathrm{rad}$. The reconstructed holograms with $\lambda_{1}=488 \mathrm{~nm}$ are shown in Figs. 4(a) and 4(b). (The lines are slightly curved because of the monitor from which the pictures were taken.) After recording the holograms, we illuminated the crystal from the other side (as shown in Fig. 1) with a $\lambda_{2}=632.8 \mathrm{~nm}$ plane wave from a helium-neon laser. Taking refraction into account ( $n=2.286$ for lithium niobate) and using the above results, we see that the estimated line separation from Eq. (3) is $\Delta x_{l}=2.4 \mathrm{~mm}$. The actual line separation was measured to be $2.18 \mathrm{~mm}$. The linewidth according to Eq. (1) is $1.9 \mathrm{~mm}$, which is approximately three times wider than the width of the lines of text.

The image reconstructed with $\lambda_{2}$ is shown in Fig. 4(c). Only one line from each of the two original images (labeled A and B in Fig. 4) appears when they are read out with the $\lambda_{2}$ plane wave (see also Ref. 13). Note that the reconstructed images shown in Figs. 4(a) and 4(b) are backward because the holograms are illuminated with the $\lambda_{2}$ and $\lambda_{1}$ beams from opposite sides of the crystal. By changing the angle of the $\lambda_{2}$ reference beam, we can reconstruct different pairs of lines from the two holograms.

By use of the above scheme an entire page of data is reconstructed simultaneously at $\lambda_{2}$; however, the stored images and the retrieved images are not the same. Since the transformation is a fixed and regular interleaving of the lines of data, we can organize the data properly so that, on retrieval, the data are organized as we desire. For instance, to store two-dimensional images directly so that they can be retrieved intact, we can preformat the data in a buffer. The preformat interleaves the images so that the subsequent shuffling at the memory readout regroups the images properly. Alternatively, the data can be interpreted at the readout end, avoiding the need for a buffer. Notice that it is possible to store one-dimensional holograms by use of onedimensional SLM's that can be completely Bragg matched. However, the storage density of the resulting hologram is drastically reduced. ${ }^{14}$

The authors thank Xin An and Michael Levene for their help.

\section{References}

1. D. L. Staebler, W. J. Burke, W. Phillips, and J. J. Amodei, Appl. Phys. Lett. 26, 182 (1975).

2. F. H. Mok, Opt. Lett. 18, 915 (1993).

3. D. Psaltis, Byte 17(9), 179 (1992).

4. F. Micheron and G. Bismuth, Appl. Phys. Lett. 20, 79 (1972).

5. Y. Qiao, S. Orlov, D. Psaltis, and R. R. Neurgaonkar, Opt. Lett. 18, 1004 (1993).

6. D. Brady, K. Hsu, and D. Psaltis, Opt. Lett. 15, 817 (1990).

7. H. Sasaki, Y. Fainman, J. E. Ford, Y. Taketomi, and S. Lee, Opt. Lett. 16, 1874 (1991).

8. S. Boj, G. Pauliat, and G. Roosen, Opt. Lett. 17, 438 (1992).

9. Q. Yong and D. Psaltis, Opt. Lett. 17, 1376 (1992).

10. S. Redfield and L. Hesselink, Opt. Lett. 13, 880 (1988).

11. R. McRuer, J. Wilde, L. Hesselink, and J. Goodman, Opt. Lett. 14, 1174 (1989).

12. G. Pauliat, J. P. Herriau, A. Delboulbe, G. Roosen, and J. P. Huignard, J. Opt. Soc. Am. B 3, 306 (1986).

13. H.-C. Külich, Appl. Opt. 30, 2850 (1991).

14. D. Psaltis, H.-Y. S. Li, and X. An, Proc. Soc. Photo-Opt. Instrum. Eng. 2026, B43 (1993). 